\title{
Community Health Collaboratives: Supporting Innovation in Public Policy, Care Delivery, and Coordination
}

\author{
Jan Nicholson, President \\ The Nicholson Foundation, Newark New Jersey
}

L IKE OTHER STATES across the nation, New Jersey is struggling with how to reduce the costs of health care while improving access and quality. In 2010, per capita health care expenditures were more than $\$ 7500$ (one of the highest levels in the United States) and $21.3 \%$ of the State's budget was devoted to Medicaid. Even with this significant spending, rates of preventable chronic diseases are high, health care access is a problem for many, and high-quality, well-coordinated care is not the norm.

In response to these realities and the opportunities afforded by the ongoing national health care reform dialogue, in 2009 The Nicholson Foundation initiated a health care grants program. Our aim was to encourage systems reform to improve the quality and reduce the costs of health care for vulnerable populations in New Jersey. We also were motivated by the realization that we could not maximize the impact of our existing programs, such as those for fragile families and disconnected and at-risk youth, without addressing access to health care and the organization and delivery of health care services.

One of our first grants in this new program area was to the Camden Coalition of Healthcare Providers. The Coalition's founders had organized a group of physicians, hospital staff, social workers, and health services organizations to address the problem of "high utilizers" in Camden by fundamentally changing the way primary health care services were delivered. When we began to work with the Camden Coalition, it had been in existence for several years but it had limited capacity and an uncertain funding base. Our support stabilized the Coalition, enhanced its infrastructure, and helped it grow and develop as an organization. It also improved the Coalition's capacity to engage in partnerships with other organizations and its ability to apply for and receive additional funding from federal agencies and other foundations. The Coalition enhanced communication, collaboration, and coordination across providers, and used data more effectively. As a result, it is now increasing the capacity and quality of primary care in Camden, expanding access to care, lowering costs, and improving outcomes.
Recognizing the value of the Camden Coalition model, we decided to sponsor its replication in several other urban centers in New Jersey. With our support, the Camden Coalition has been providing technical assistance to strengthen an existing community-led coalition in Trenton-the Trenton Health Team-and to help Newark and its surrounding region develop the Greater Newark Healthcare Coalition. We also have provided grants and other support directly to the Trenton and Newark Coalitions.

Our work with these Coalitions shows that foundations can play a critical role in seeding innovation and helping good ideas and programs come to fruition. However, we also are interested in sustainability. Reforming the organization and delivery of health care services for vulnerable populations will not ultimately be successful unless access to stable, long-term funding is available. In 2010, the Coalitions, in partnership with community leaders and other stakeholders, approached State policy makers to explore potential strategies for developing a new organizational design that would not only improve the delivery of health care services to vulnerable populations in New Jersey, but also provide a sustainable funding stream for these efforts. The result was the passage of the Medicaid Accountable Care Organization (ACO) Demonstration Project in August 2011.

New Jersey's Medicaid ACOs are different from Medicare or private ACOs because they are responsible for improving health care quality and reducing its costs for all Medicaid patients in an ACO region, not just the patients of specific providers or hospitals. Moreover, to ensure broad community involvement, a Medicaid ACO requires that all the hospitals and nearly all of the other health care providers in the region actively participate.

Medicaid ACOs have considerable potential to improve the health of vulnerable populations, but their development is complex and challenging. It is therefore important that all those involved have the knowledge and ability to participate successfully. In describing the unique characteristics of the New Jersey Medicaid ACO model and exploring the

The Nicholson Foundation works to address the complex needs of vulnerable populations in New Jersey's urban areas by encouraging the reform of health and human services delivery systems. Our approach emphasizes partnerships and performance-based grant making; our aims are sustainability and systems reform. For more information about the Foundation, please visit http://www.thenicholsonfoundationnewjersey.org. 
history and development of the Camden, Trenton, and Newark Coalitions, this supplement complements our ongoing ACO training and technical assistance activities. These include supporting workshops and conferences for stakeholders, funding the development of the New Jersey Medicaid Accountable Care Organization Business Planning Toolkit, and sponsoring an ACO learning network coordinated by the New Jersey Health Care Quality Institute.

The Nicholson Foundation is very pleased to support this special supplement to Population Health Management. We are optimistic that it will encourage other urban areas to consider developing community-led Medicaid ACOs. We also hope that it stimulates government and others to provide the resources necessary to expand and strengthen this innova- tive model of health care delivery. Improving health care quality and reducing costs is a national imperative, and we are pleased to contribute to the development of long-term, effective solutions.

Address correspondence to: Barbara Kang

Director of Community Health

The Nicholson Foundation

744 Broad St

26th floor

Newark, NJ 07102-3802

E-mail: barbarahkang@gmail.com 